A Prototype of the SNS Optics Database

BNL/SNS TECHNICAL NOTE

NO. 085

Nikolay Malitsky

November 9, 2000

COLLIDER-ACCELERATOR DEPARTMENT

BROOKHAVEN NATIONAL LABORATORY

UPTON, NEW YORK 11973 


\title{
A Prototype of the SNS Optics Database.
}

\author{
Nikolay Malitsky
}

\section{Introduction}

Database is an important part of the modern software environment. It aims to consolidate diverse data sets and facilitate their analysis and management. Accelerator data can be distributed into several domain databases, such as Naming, Survey, Magnet, Optics, and others. Each domain is maintained by the corresponding group of specialists and is associated with their particular applications. The Optics database is a persistent representation of the accelerator model and provides accelerator physicists with data of " all elements that can influence single particle motion (in their idealized operation) and only those elements ... "[1]. Usually, the schema of the Optics database reflects the structure of existing accelerator codes. In 1992, the LAMBDA collaboration [2] had introduced the most complete implementation of the Optics database employing the MAD model. Since that time, a new generation of object-oriented accelerator packages has been introduced and developed. For the SNS ring applications, we are using the Unified Accelerator Libraries (UAL) environment [3] that currently joins together six object-oriented libraries, such as PAC, TEAPOT, ZLIB, ACCSIM, ORBIT, and ALE. The paper presents a version of the Optics database representing the UAL accelerator model.

\section{Schema of the Optics Database}

The schema of the Optics database has been implemented after the UAL accelerator object model using the object-relational mapping approach [4]. Figure 1 illustrates the structure of the UAL accelerator model:

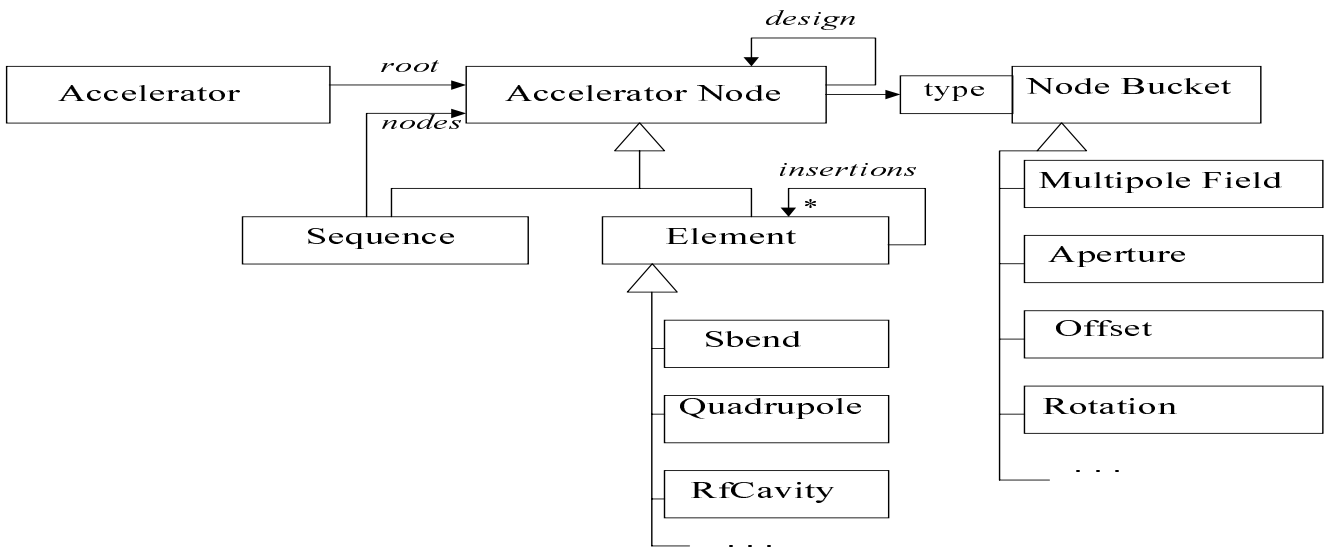

Figure 1: UAL accelerator object model. 
The model is based on three entities:

- Accelerator is a hierarchical tree of accelerator nodes, elements and sequences of elements.

- Accelerator Node is a basis class of an accelerator element and sequence of elements. There are many different types of accelerator nodes (e.g. sbend, quadrupole, etc.) But all of them have the same structure: an open collection of accelerator node buckets.

- Accelerator Node Bucket is a set of attributes relevant to the single physical effect or feature (e.g. magnetic field, aperture, offset, rotation, etc.)

The following sections describe the representation of these objects in the Optics database.

\subsection{Accelerator}

In the UAL model, an accelerator is described as a hierarchical tree of accelerator nodes. This definition is very general and can be applied to different accelerator machines, their components, or transfer lines. Usually, the modern accelerator complexes contain several accelerators and each of them is developed by a separate group of scientists. To avoid naming collisions among different teams, each accelerator identifier determines a separate naming space for its elements and is used as the first component of combine element names in the Optics Database. All accelerator identifiers are located in the following table:

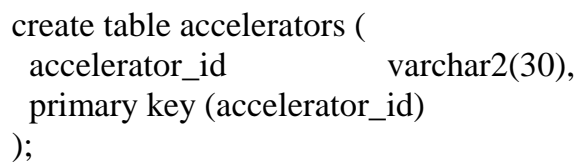

The UAL accelerator description is highly modularized. An accelerator structure is composed of independent entities, accelerator nodes. Each accelerator node, in turn, is built from the collection of separate element buckets, orthogonal sets of element attributes. Such organization facilitates a fine-grain approach for the versioning of accelerator data. In our scheme, the accelerator description has three independent version attributes: lattice_id, state_id, and source_id. The first attribute is associated with an accelerator structure. The state_id attribute represents a set of element buckets used in the particular application; source_id defines the origin of element data (e.g. expectation, measurement, etc.). In the Optics database, each of these attributes has its own table:

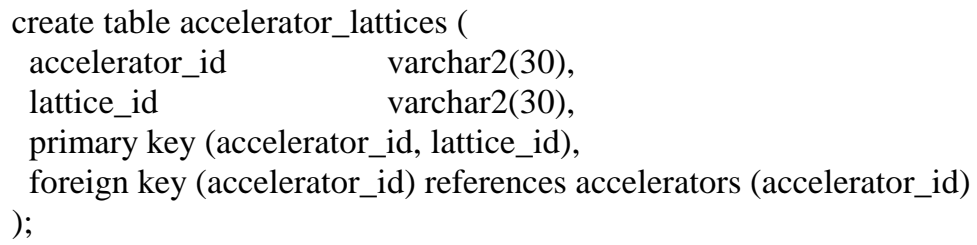




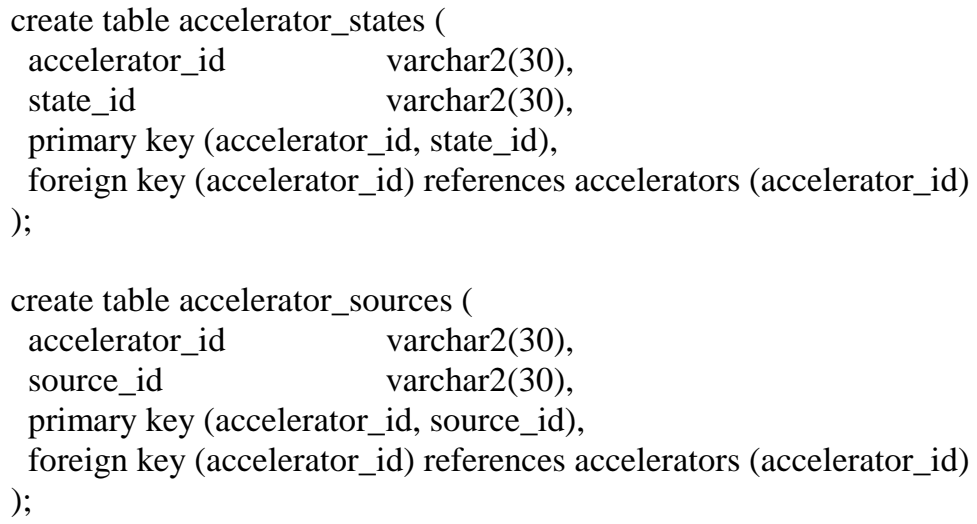

A set of accelerators together with their version attributes is associated with system_id. All these associations are stored in the accelerator_systems table:

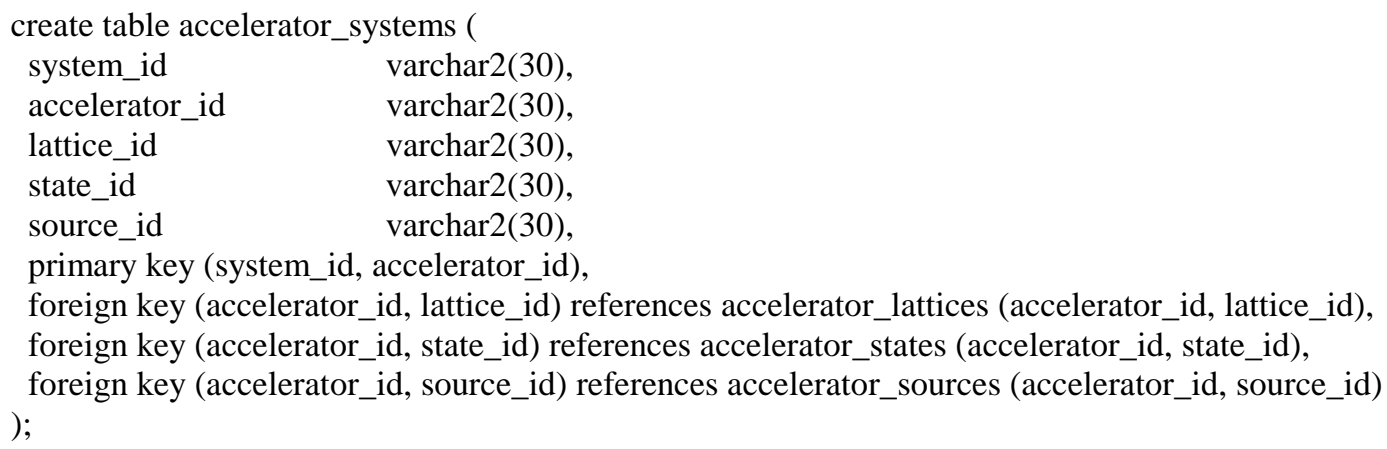

\subsection{Accelerator Node}

Accelerator node is a basis class of various types of accelerator elements and sequences of elements. In the Optics database, this class is represented by the corresponding table:

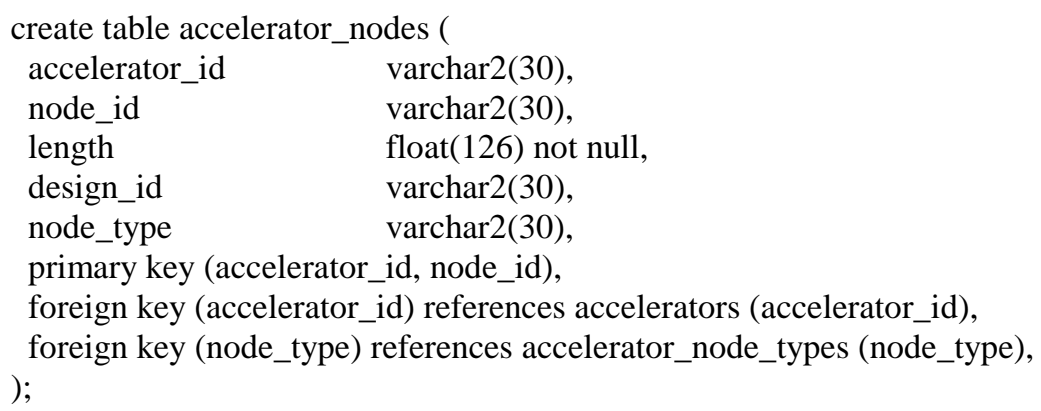

The first two fields, node_id and accelerator_id, form a composite element identifier that provides the referential integrity of the whole Optics database. The third field is an effective length of the accelerator node. The optional attribute design_id is a reference to the design element. This reference allows one to support the relationship between 
operational and design accelerator descriptions. The discriminator node_type specifies the type of the accelerator node.

The UAL model preserves all MAD element types (such as sbend, quadrupole, rfcavity, etc.) and defines the mechanism for introducing new ones. A predefined set of legal UAL node types is located in the accelerator_node_types table:

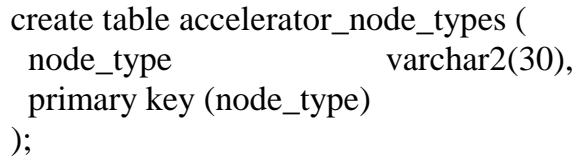

Elements of different types have the same structure and do not require to be represented by specialized tables. Contrary, the Sequence, a composite node in an accelerator hierarchical tree, introduces an one-to-many relationship between composite and contained nodes. According to the object-relational mapping approach, this association is implemented in the following table:

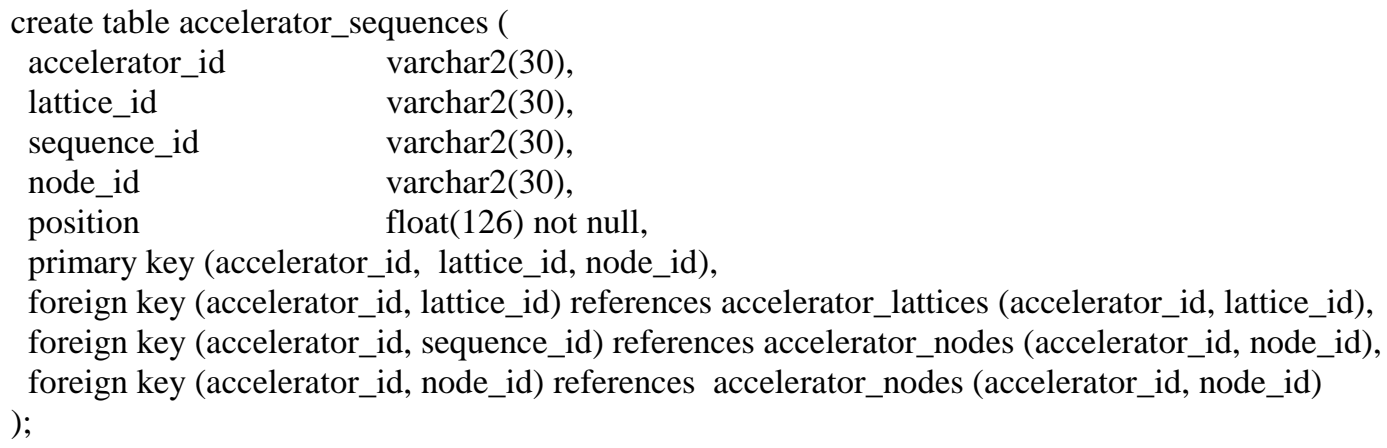

The accelerator_id and lattice_id fields specify the accelerator name and the version of the accelerator structure, respectively. The node_id column keeps identifiers of the accelerator nodes contained into the sequence_id structure. The position attribute is a longitudinal position of the node with respect to the beginning of the parent sequence.

\subsection{Accelerator Node Bucket}

Accelerator node bucket encapsulates the minimal set of attributes relevant to the single physical effect (e.g. magnetic field, offset), element feature (e.g. size, aperture), or special algorithm parameters. This structure addresses several tasks. First, it facilitates selection and classification of well-defined concrete data types. Second, it provides a consistent scalable approach for introducing and integrating new elements and element attributes. Finally, it offers a uniform algorithm-neutral element description that is open to diverse applications. Usually, each application requires an individual combination of element buckets. For example: a survey layout is based only on geometrical parameters; design programs use main element attributes; simulation codes includes field errors, misalignments, aperture, and other features that are critical for the particular scenario and accelerator. These applications can be configured in many different ways. In the Optics database, all these configurations are stored in the accelerator_node_buckets table: 


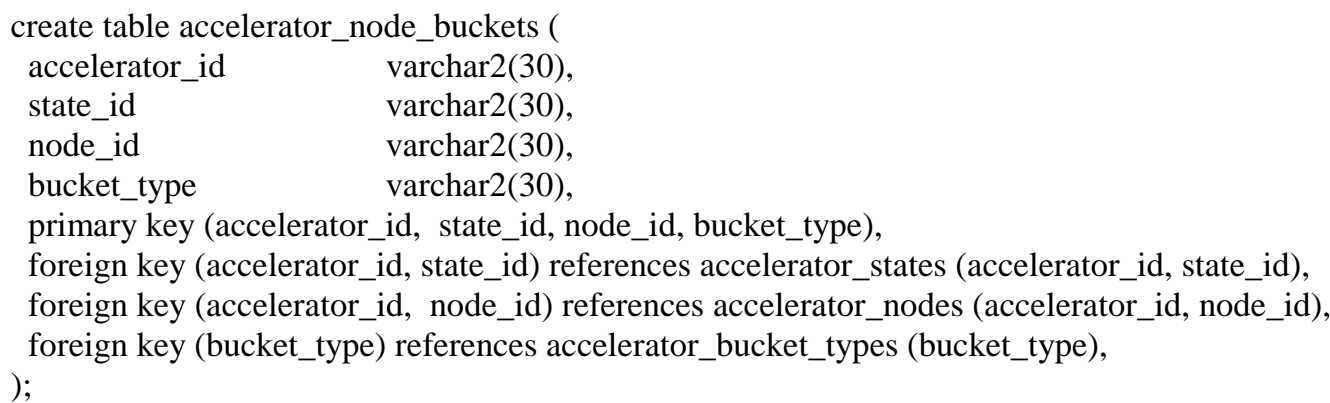

The accelerator_id and state_id fields specify the accelerator name and the version of the accelerator node configuration, respectively. The bucket_type column keeps identifiers of buckets included in the node_id structure.

A predefined set of legal UAL bucket types is located in the accelerator_bucket_types table:

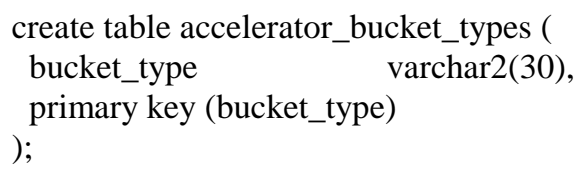

Each bucket type is represented by the separate table. At this time, we consider buckets with bend attributes, magnet and accelerating fields, alignment errors, and aperture parameters:

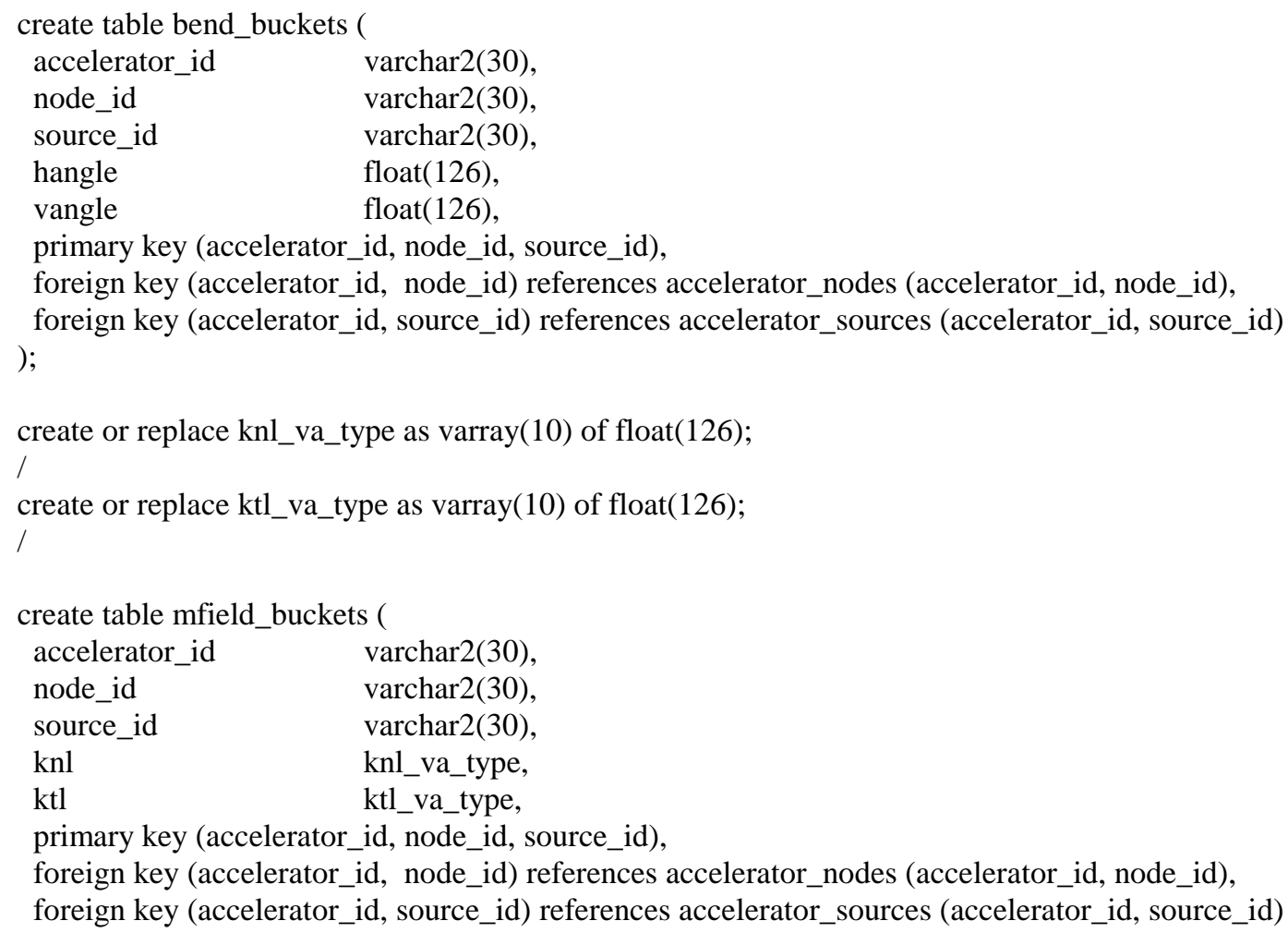




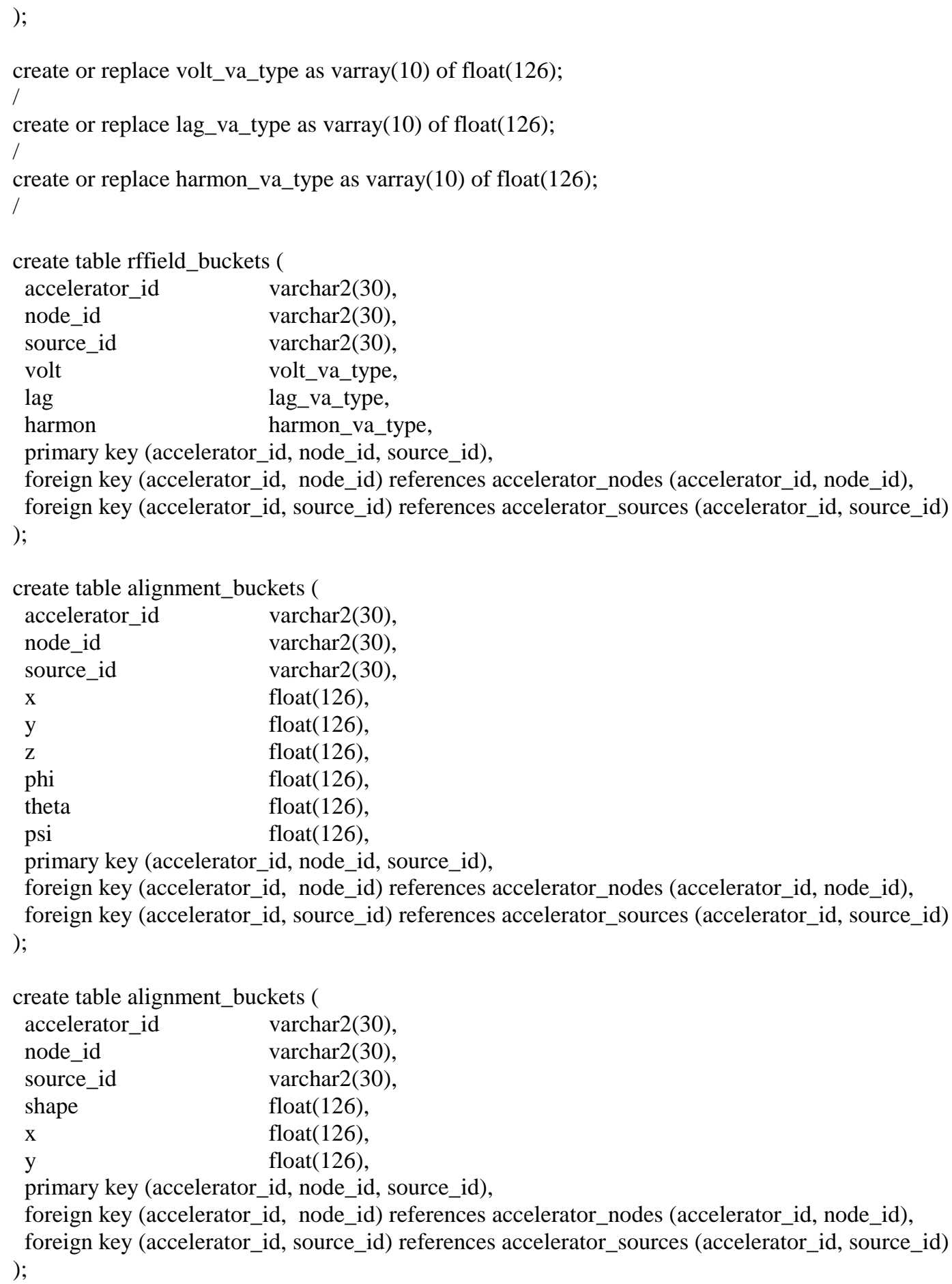

All these tables have a similar structure. The first two fields, accelerator_id and node_id, identify the accelerator node. The source_id field is associated with the origin of bucket attributes. Attribute values can be initialized from many different sources: physicist's assumptions and requirements, calculation, or other specialized databases (such as Magnet and Survey databases). The list of other columns is summarized in the table 1: 
Table 1 : List of bucket tables.

\begin{tabular}{|c|c|c|c|}
\hline $\begin{array}{l}\text { Bucket } \\
\text { type }\end{array}$ & Table & Columns & Description \\
\hline \multirow[t]{2}{*}{ bend } & \multirow[t]{2}{*}{ bend_buckets } & hangle & horizontal bend angle \\
\hline & & vangle & vertical bend angle \\
\hline \multirow[t]{2}{*}{ mfield } & \multirow[t]{2}{*}{ mfield_buckets } & $\mathrm{knl}$ & array of normal multipole components \\
\hline & & ktl & array of skew multipole components \\
\hline \multirow[t]{3}{*}{ rffield } & \multirow[t]{3}{*}{ rffield_buckets } & volt & array of RF voltages \\
\hline & & lag & array of phase lags \\
\hline & & harmon & array of harmonic numbers \\
\hline \multirow[t]{6}{*}{ alignment } & \multirow[t]{6}{*}{ alignment_buckets } & $\mathrm{x}$ & offset in the $\mathrm{x}$-direction \\
\hline & & $\mathrm{y}$ & offset in the $y$-direction \\
\hline & & $\mathrm{Z}$ & offset in the $\mathrm{z}$-direction \\
\hline & & phi & rotation around the $\mathrm{x}$-axis \\
\hline & & theta & rotation around the $y$-axis \\
\hline & & psi & rotation around the s-axis \\
\hline \multirow[t]{3}{*}{ aperture } & \multirow[t]{3}{*}{ aperture_buckets } & shape & aperture shape \\
\hline & & $\mathrm{x}$ & horizontal half-aperture \\
\hline & & $\mathrm{y}$ & vertical half-aperture \\
\hline
\end{tabular}

\section{Future Activities}

The presented schema defines a frame of the Optics database. Currently, we are working on a mechanism for the initialization of its tables. Data can be inserted into the database in many different ways. In the past, each vendor offered the specialized user-friendly environment that facilitated access to the database records (e.g. Oracle Forms). The choice of the particular vendor or tool was very important and determined the structure of project applications. At this time, the Web technologies have changed the situation by defining several industrial standards for each layer of database-based multi-tier infrastructure. It shifts the emphasis in the software development process from the selection of the particular tool to the selection of the most appropriate and promising industrial standards. The essential part of the modern infrastructure is occupied by the Extensible Markup Language (XML) technology. XML has become very popular in many areas and is considered as a universal mechanism for the initialization and configuration of various software systems. In our environment, we plan to employ the XML software for integrating off-line and online simulation facilities and developing interfaces based on Accelerator Description Exchange Format (ADXF [5]) files (see Fig. 2). ADXF was developed to provide a complete and uniform description of accelerator data used in diverse beam dynamics programs. The structure of this description is mapped from the common UAL accelerator object model and theoretically consistent with other accelerator model representations. Our next application will integrate together three representations: Accelerator Model classes, Optics database, and ADXF files. It 
allows us to test the compatibility of their structures and initialize the Optics database tables from the ADXF file.

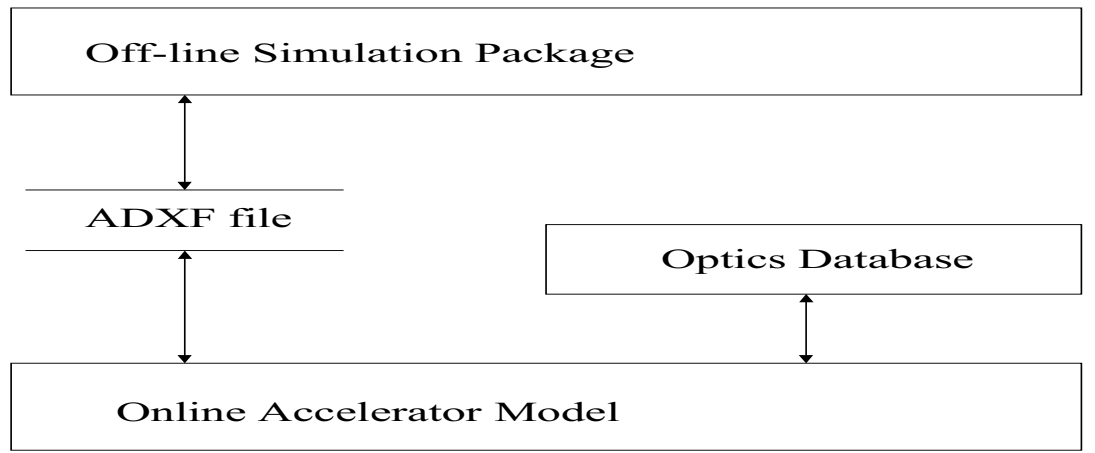

Figure 2 : ADXF-based interfaces.

\section{Acknowledgments}

The author would like to thank T. Nepsee, S.Sathe, J.Smith, and J.Wei for many valuable discussions.

\section{References:}

[1] F.C.Iselin, E.Keil, R.Talman. Request for the Accelerator Description Standard. 1998.

[2] S.Peggs et al. LAMBDA Manual. RHIC/AP/13, 1993.

[3] N.Malitsky and R.Talman. Unified Accelerator Libraries. CAP, 1996.

[4] M.Blaha and W.Premerlani. Object-Oriented Modeling and Design for Database

Applications. Prentice Hall, 1998.

[5] N.Malitsky and R.Talman. Accelerator Description Exchange Format, ICAP, 1998. 